\title{
Testing the existence of optical linear polarization in young brown dwarfs
}

\author{
E. Manjavacas, ${ }^{1,2,3 \star}$ P. A. Miles-Páez, ${ }^{1,2,4}$ M. R. Zapatero-Osorio, ${ }^{5}$ B. Goldman,,${ }^{6,7}$ \\ E. Buenzli, ${ }^{8}$ T. Henning, ${ }^{7}$ E. Pallé ${ }^{1,2}$ and M. Fang ${ }^{3}$ \\ ${ }^{1}$ Instituto de Astrofísica de Canarias, C/ Vía Láctea, s/n, E38205 - La Laguna (Tenerife), Spain \\ ${ }^{2}$ Dpt. de Astrofísica, Univ. de La Laguna, Avda. Astrofísico Francisco Sánchez s/n, E-38206 La Laguna (Tenerife), Spain \\ ${ }^{3}$ Department of Astronomy/Steward Observatory, The University of Arizona, 933 N. Cherry Avenue, Tucson, AZ 85721, USA \\ ${ }^{4}$ The University of Western Ontario, Department of Physics and Astronomy, 1151 Richmond Avenue, London, ON N6A 3K7, Canada \\ ${ }^{5}$ Centro de Astrobiología (CSIC-INTA), Carretera de Ajalvir km 4, E-28850 Torrejón de Ardoz, Madrid, Spain \\ ${ }^{6}$ Observatoire astronomique de Strasbourg, Université de Strasbourg, CNRS, UMR 7550, 11 rue de l'Université, F-67000 Strasbourg, France \\ ${ }^{7}$ Max-Planck-Institut für Astronomie, Königstuhl, 17. D-69117 Heidelberg, Germany \\ ${ }^{8}$ Institute for Astronomy, ETH Zurich, Wolfgang-Pauli-Strasse 27, CH-8093 Zurich, Switzerland
}

Accepted 2017 March 20. Received 2017 March 13; in original form 2016 December 8

\begin{abstract}
Linear polarization can be used as a probe of the existence of atmospheric condensates in ultracool dwarfs. Models predict that the observed linear polarization increases with the degree of oblateness, which is inversely proportional to the surface gravity. We aimed to test the existence of optical linear polarization in a sample of bright young brown dwarfs, with spectral types between M6 and L2, observable from the Calar Alto Observatory, and cataloged previously as low gravity objects using spectroscopy. Linear polarimetric images were collected in $I$ and $R$ band using CAFOS at the 2.2-m telescope in Calar Alto Observatory (Spain). The flux ratio method was employed to determine the linear polarization degrees. With a confidence of $3 \sigma$, our data indicate that all targets have a linear polarimetry degree in average below 0.69 per cent in the $I$ band, and below 1.0 per cent in the $R$ band, at the time they were observed. We detected significant (i.e. $P / \sigma \geq 3$ ) linear polarization for the young M6 dwarf 2MASS J04221413+1530525 in the $R$ band, with a degree of $p^{*}=0.81 \pm 0.17$ per cent.
\end{abstract}

Key words: brown dwarfs - stars: low-mass - infrared: stars.

\section{INTRODUCTION}

Brown dwarfs are sub-stellar objects that do not have enough mass to maintain stable hydrogen fusion in their cores, therefore, brown dwarfs cool with time, contracting and changing spectral types, from the M to Y spectral class (Kirkpatrick et al. 2012). Due to their evolution, the age and the mass are not determined by the spectral type, in contrast to stars. Thus, in addition to a spectrum, an accurate determination of brown dwarf ages is necessary to determine stellar masses (Burrows et al. 1997).

Young brown dwarfs, with ages $\leq 500 \mathrm{Myr}$, are still contracting to their final radii. Therefore, young brown dwarfs have lower gravity than mature field brown dwarfs ( $>500 \mathrm{Myr}$ ). Low gravity affects the chemistry of the atmosphere of brown dwarfs, leading to the modification of some of the spectral features present in more evolved brown dwarfs: they have weaker alkali lines, and in some cases redder colours and a triangular $H$ band (Lucas et al. 2001; Gorlova et al. 2003). There are also very red low mass dwarfs that do not show any evidence of youth or low gravity atmospheres

^E-mail: elenamanjavacas@email.arizona.edu
(Marocco et al. 2014; Liu, Dupuy \& Allers 2016). It has been suggested that the infrared flux excesses observed in both lowand high-gravity very red cool dwarfs are caused by extraordinary dusty atmospheres (Looper et al. 2008; Barman et al. 2011; Gizis et al. 2012; Allers et al. 2016), although other scenarios might be at play (e.g. different metallicity, presence of discs).

Linear polarization is likely produced by scattering processes of the dusty particles in brown dwarfs atmospheres (Sengupta \& Krishan 2001; Sengupta 2003; Sengupta \& Kwok 2005). Polarization can thus become a useful method to determine the properties of the atmospheric dust as the degree of polarization correlates with the particle size (Sengupta \& Krishan 2001). Sengupta \& Marley (2010, and references therein) predicted optical and near-infrared values of linear polarization in the range $0-1$ per cent for dusty ultracool dwarfs. Non-zero linear polarization due to scattering processes is thought to arise as a result of the non-cancellation of the polarization signal from different areas of the ultracool dwarf due to asymmestries such as an oblate shape or an heterogeneous distribution of dust. At lower gravities, rotationally induced non-sphericity is favoured. Therefore, for ultracool dwarfs with similar amounts of condensates, we expect that the degree of linear polarization increases with the degree of oblatness. Furthermore, Marocco et al. 
(2014) and Hiranaka et al. (2016) concluded that under the scenario of a dust haze of particles in the upper atmospheres, the grains must have sub-micron sizes (typically between 0.15 and $0.4 \mu \mathrm{m}$ ) in order to explain the observed red colours.

Observationally, linear polarization in the optical and in the nearinfrared has been detected for some mature ultracool dwarfs, and also for young brown dwarfs (Ménard, Delfosse \& Monin 2002; Zapatero Osorio, Caballero \& Béjar 2005; Goldman et al. 2009; Tata et al. 2009; Zapatero Osorio et al. 2011; Miles-Páez et al. 2013; Miles-Páez, Zapatero Osorio \& Pallé 2015). The degree of polarization measured in field brown dwarfs in the optical is up to $\sim 1.4$ per cent, which agrees with the theoretical predictions.

In this paper, we present the linear polarimetric photometry of a sample of well-selected low-gravity dwarfs whose masses are quite likely sub-stellar. By comparing our measurements with those of high-gravity dwarfs of related spectral types available in the literature, we will test whether there is a relation between linear polarization intensity and surface gravity.

\section{SAMPLE SELECTION}

We selected ultracool dwarfs from Allers \& Liu (2013), with spectral types in the M-L transition (M6-L2), and confirmed as young objects, i.e. as low gravity brown dwarfs. All targets were observable from the Calar Alto observatory (Spain), and were bright enough $(J \leq 13.5 \mathrm{mag}$ ) in the $R$ and $I$ bands to achieve a precision of $\sigma_{P} \pm 0.2$ percent with a 2.2-m telescope. Only six objects of Allers \& Liu (2013) sample satisfied our selection criteria. In Table 1, we provide a list of the targets with their magnitudes in $J$ and $W 2$ bands, their spectral types, distances and their gravity flags. Henceforth, we will use abridged names.

Our targets are brown dwarfs with low gravity signatures in their spectra: weak alkali metal lines, triangular $H$ band and redder colours than field brown dwarfs with the same spectral type. These objects have estimated ages lower than $\sim 500 \mathrm{Myr}$.

$2 \mathrm{M} \mathrm{J} 0045+1634$ is classified as an L0 $\beta$ by Faherty et al. (2016), has lithium in its atmosphere and is a likely member of the Argus moving group according to Zapatero Osorio et al. (2014). Its age is estimated at the interval 10-100 Myr. Allers \& Liu (2013) classified this object as a very low gravity dwarf using near-infrared spectra, which is consistent with a young age. Both Miles-Páez et al. (2013) and Zapatero Osorio et al. (2005) measured very low linear polarization indices, compatible with null polarization and in any case below 0.12 per cent, in the $J$ and $I$ bands.

Object 2M J0335+2342 is a M8.5 dwarf with Li detection at $6708 \AA$ (Reid et al. 2002), which provides an upper limit on its age of $\sim 150$ Myr for $\mathrm{M}$ dwarfs. It has intense and resolved $\mathrm{H} \alpha$ emission as well (Shkolnik, Liu \& Reid 2009). Allers \& Liu (2013) re-classified it as a young M7. Gagné et al. (2014) discussed that it might be a likely member of the $\beta$-Pictoris moving group.

The object $2 \mathrm{M} \mathrm{J} 0422+1530$ was classified as a M6 $\gamma$ by Cruz, Kirkpatrick \& Burgasser (2009) using its optical spectra. Allers \& Liu (2013) found that its near-infrared spectrum was excessively red for its spectral type, and showed weak alkali metal lines, indicating low surface gravity. Faherty et al. (2012) reported an absolute parallax of $24.8 \pm 3.1$ mas $(\mathrm{d}=40.3 \pm 5.0 \mathrm{pc})$, which is $6.4 \sigma$ discrepant with the absolute parallax reported by Liu et al. (2016) $\left(\pi_{\mathrm{abs}}=3.9 \pm 1\right.$ mas or $d=240_{-40}^{+70} \mathrm{pc}$ ). $2 \mathrm{M} 0422$ is $\sim 10^{\circ}$ to the South of the Taurus star-forming region on the sky (age $\sim 1 \mathrm{Myr}$, Briceño et al. 1999; Luhman 2004), therefore, depending on which of the two values of the trigonometric distance reported is correct, 2M0422 might be before, embedded or behind the Taurus-Auriga star-forming region.

$2 \mathrm{M} \mathrm{J} 0443+0002$ is a L0 dwarf classified as a very low gravity object by Allers \& Liu (2013), showing all typical spectral characteristics of a low gravity dwarf.

$2 \mathrm{M} \mathrm{J0602+3910} \mathrm{is} \mathrm{a} \mathrm{L2} \mathrm{intermediate} \mathrm{gravity} \mathrm{object} \mathrm{(Allers} \mathrm{\&}$ Liu 2013), and a candidate member to the Pleiades moving group, with an estimated age of $\sim 100 \mathrm{Myr}$ (Seifahrt et al. 2010).

Finally, the object $2 \mathrm{M} \mathrm{J} 2057-0252$ is a L2 intermediate gravity brown dwarf based on its low-resolution spectrum (Allers \& Liu 2013). Its optical spectrum shows both lithium absorption and $\mathrm{H} \alpha$ emission (Cruz et al. 2003). Zapatero Osorio et al. (2005) measured its linear polarization in $I$ band obtaining a debiased value of $p^{*}=0.00 \pm 0.38$ per cent, and Miles-Páez et al. (2013) obtained a polarization in the $J$ band of $p^{*}=0.13 \pm 0.15$ per cent.

\section{OBSERVATIONS AND DATA REDUCTION}

We collected linear polarimetric images using the Calar Alto Faint Object Spectrograph (CAFOS, Patat \& Taubenberger 2011), at the 2.2-m telescope at the Calar Alto Observatory (Spain). It is mounted at the Cassegrain focus, and it is equipped with a Wollaston prism for polarimetry that provides an effective beam separation of $\sim 20$ arcsec, plus a half-wave retarder plate. With this combination, we were able to measure linear polarization using dual-beam imaging polarimetry. CAFOS has a $2048 \times 2048$ pixels SITe CCD detector, with a scale of $0.53 \operatorname{arcsec}_{\text {pixel }}{ }^{-1}$. The CCD was windowed to the central $1024 \times 1024$ pixels, with a field of view of $9 \times 9 \operatorname{arcsec}^{2}$.

Table 1. List of observed targets with their magnitudes, spectral types and gravity characteristics.

\begin{tabular}{|c|c|c|c|c|c|c|}
\hline Name & $J$ (mag) & $W 2(\mathrm{mag})$ & $d(\mathrm{pc})$ & $\mathrm{SpT}$ & Gravity flag $^{a}$ & Reference \\
\hline 2MASS J03350208+2342356 & $12.25 \pm 0.02$ & $10.77 \pm 0.02$ & $42.4 \pm 2.3$ & M7 & VL-G, $10 \mathrm{Myr}$, Li detection [7] & 2,7 \\
\hline & & & $240_{-40}^{+70}$ & M6 $\gamma$ & VL-G & 3,11 \\
\hline 2MASS J04433761+0002051 & $12.51 \pm 0.03$ & $10.48 \pm 0.02$ & & L0 & VL-G & 4 \\
\hline 2MASS J06023045+3910592 & $12.30 \pm 0.02$ & $10.13 \pm 0.02$ & & L2 & INT-G, $\sim 100$ Myr [10] & 5 \\
\hline
\end{tabular}

References: [1] - Kendall et al. (2004), [2] - Gizis et al. (2000), [3] - Reid, Hawley \& Gizis (1995), [4] - Hawley et al. (2002), [5] - Lépine, Shara \& Rich (2002), [6] - Ménard et al. (2002), [7] - Shkolnik et al. (2012), [8] - Faherty et al. (2012), [9] - Zapatero Osorio et al. (2014), [10] - Allers \& Liu (2013), [11] - Liu et al. (2016)

${ }^{a}$ Gravity class classification provided by Allers \& Liu (2013): VL-G: the object has spectral characteristics consistent with very low gravity; INT-G: the object has spectral characteristics consistent with intermediate gravity dwarfs. 
Observations were obtained during the whole nights between 2014 October 23 and 27.

Images were obtained in $R(641 \mathrm{~nm}$, passband $158 \mathrm{~nm})$ and $I$ bands $(850 \mathrm{~nm}$, passband $150 \mathrm{~nm})$. All targets were observed in both filters in different days. Bias calibration frames, and twilight and dome flat frames with the polarization optics were taken every day in $I$ and $R$ filters. No systematic variation was detected in the calibration frames from night to night. Beside our targets, we observed two types of standard stars at approximately the same CCD spot $(512,512$ on the windowed images): two polarized standard stars, BD+25 727 and HD251204, and two non-polarized standard stars, G191B2B and BD+28 4211. To investigate instrumental polarization, we used only G191B2B because the other unpolarized star has a close visual companion that may affect the polarization measurements. The white dwarf G191B2B allowed us to test the stability of the instrument and the existence of instrumental polarization, while the polarized standard stars allow us to identify zero offsets.

We collected images at four different angles of the retarder plate: $0^{\circ}, 22.5^{\circ}, 45^{\circ}$ and 67.5 . The observing log is shown in Table A1, including date of observation for each target, filter of observation, exposure time, number of exposures per each retarder plates position, airmass, aperture and full width at high maximum (FWHM).

We reduced the raw images using the IRAF (Image Reduction and Analysis Facility) standard routines. Raw images were biassubstracted and flat-field corrected using the corresponding images to their respective filter. We used the dome flats acquired as a part of the daily standard calibrations taken in the telescope.

\section{POLARIMETRIC ANALYSIS}

We calculated the degree of linear polarization in the $I$ and $R$ bands, and the polarization angles by means of the Stokes parameters $q$ and $u$. The equations to compute the Stokes parameters, the degree of linear polarization and the angle of vibration of the polarization are taken from Zapatero Osorio et al. (2011) and Miles-Páez et al. (2013):

$R_{q}^{2}=\frac{o(0) / e(0)}{o(45) / e(45)}$

$R_{u}^{2}=\frac{o(22.5) / 22.5(0)}{o(67.5) / e(67.5)}$,

$q=\frac{R_{q}-1}{R_{q}+1}$,

$u=\frac{R_{u}-1}{R_{u}+1}$,

$P=\sqrt{q^{2}+u^{2}}$,

$\theta=0.5 \tan ^{-1}(u / q)$,

where $o$ refers to the flux of the ordinary beam, and $e$ refers to the flux of the extraordinary beam in the dual images of the single frames. $P$ and $\theta$ are the linear polarization and the angle of vibration of the linear polarization, respectively.

Fluxes of the targets and reference stars have been derived by doing aperture photometry using different aperture radii and different annuli for the sky at different distances from the centroid of the target. We calculated the flux for every target employing circular photometric apertures of different sizes, from 0.5-6 $\times$ FWHM with steps of $0.1 \times$ FWHM, and six sky rings, which were annuli with

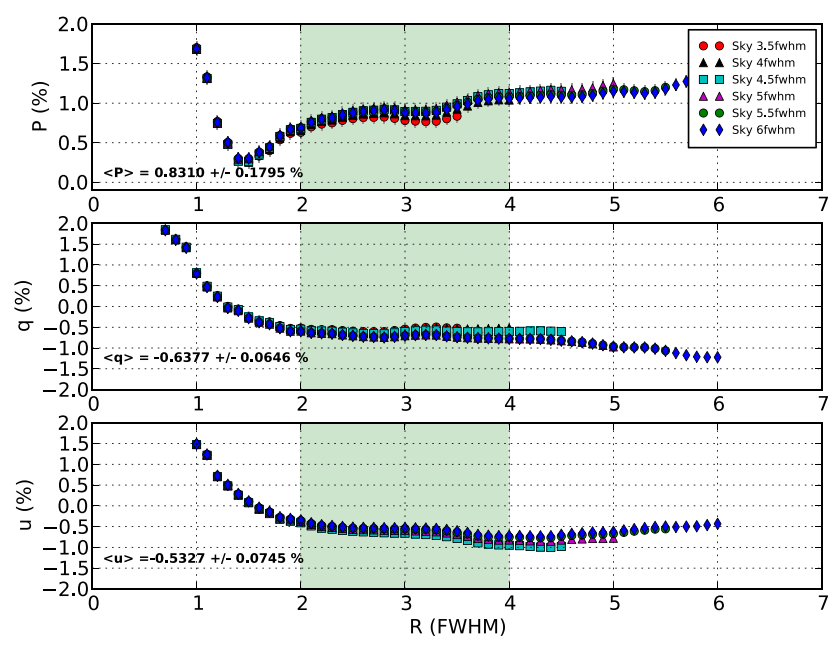

Figure 1. Normalized Stokes parameters $q$ (middle) and $u$ (bottom), and the degree of linear polarization $P$ in the $R$ band as a function of the aperture radius (in FWHM unit) for the polarized object 2M0422. The green area denotes the region for which we averaged the Stokes parameters $q$ and $u$ (mean values also shown in each panel).

inner radii of 3.5 through $6 \times$ FWHM (steps of $0.5 \times$ FWHM) and widths of $1 \times$ FWHM. These fluxes were used to compute the Stokes parameters, $q$ and $u$. We chose the range of apertures in which their estimated values remained nearly flat (typically $2-5 \times$ FWHM, depending on brightness and filter, see Table A1). For every night and target, we obtained figures similar to Fig. 1. All the measurements are compatible with zero polarization within $3 \sigma$, suggesting that the instrument is free of instrumental polarization. The error obtained in the $I$ band for the unpolarized stars was 0.17 per cent, and for the $R$ band was 0.18 per cent. Uncertainties $u$ and $q$ are estimated as the standard deviation of values of the Stokes parameters $u$ and $q$. Uncertainties in $P$ are the quadratic sum of the uncertainties in $q$ and $u$, and the uncertainty derived from non-polarized standard star ( 0.17 per cent for the $I$ band and 0.18 per cent for the $R$ band). The error in the polarization vibration angle is

$\sigma_{\theta}=28.65 \sigma_{\mathrm{P}} / P$

where $\sigma_{\theta}$ is the error of the linear polarization angle in degrees, $\sigma_{\mathrm{P}}$ is the uncertainty of the polarization degree. The factor $28.65 \sigma_{\mathrm{P}} / P$ comes from the propagation of uncertainties, and it is only valid when $P / \sigma_{\mathrm{P}} \geq 3$ (Serkowski 1974; Wardle \& Kronberg 1974). We identified as linearly polarized those sources in our sample that comply with $P / \sigma_{\mathrm{P}} \geq 3$ (i.e. $3 \sigma$ criterion).

The degree of linear polarization is always a positive quantity, thus, small values of $P$ and measurements affected by poor $\mathrm{S} / \mathrm{N}$ are biased towards an overestimation of the true polarization. We applied Wardle \& Kronberg (1974) equation to debiase the linear polarization degree as follows:

$p *=\sqrt{P^{2}-\sigma_{\mathrm{P}}^{2}}$.

We provide the observation dates, filter, Stokes parameters, linear polarization degree and debiased linear polarization degree with their respective uncertainties in Table 2. Polarization vibration angles are only provided for those sources that satisfy the $3 \sigma$ criterion, and therefore are likely linearly polarized. 
Table 2. Linear polarimetric results of the observed targets.

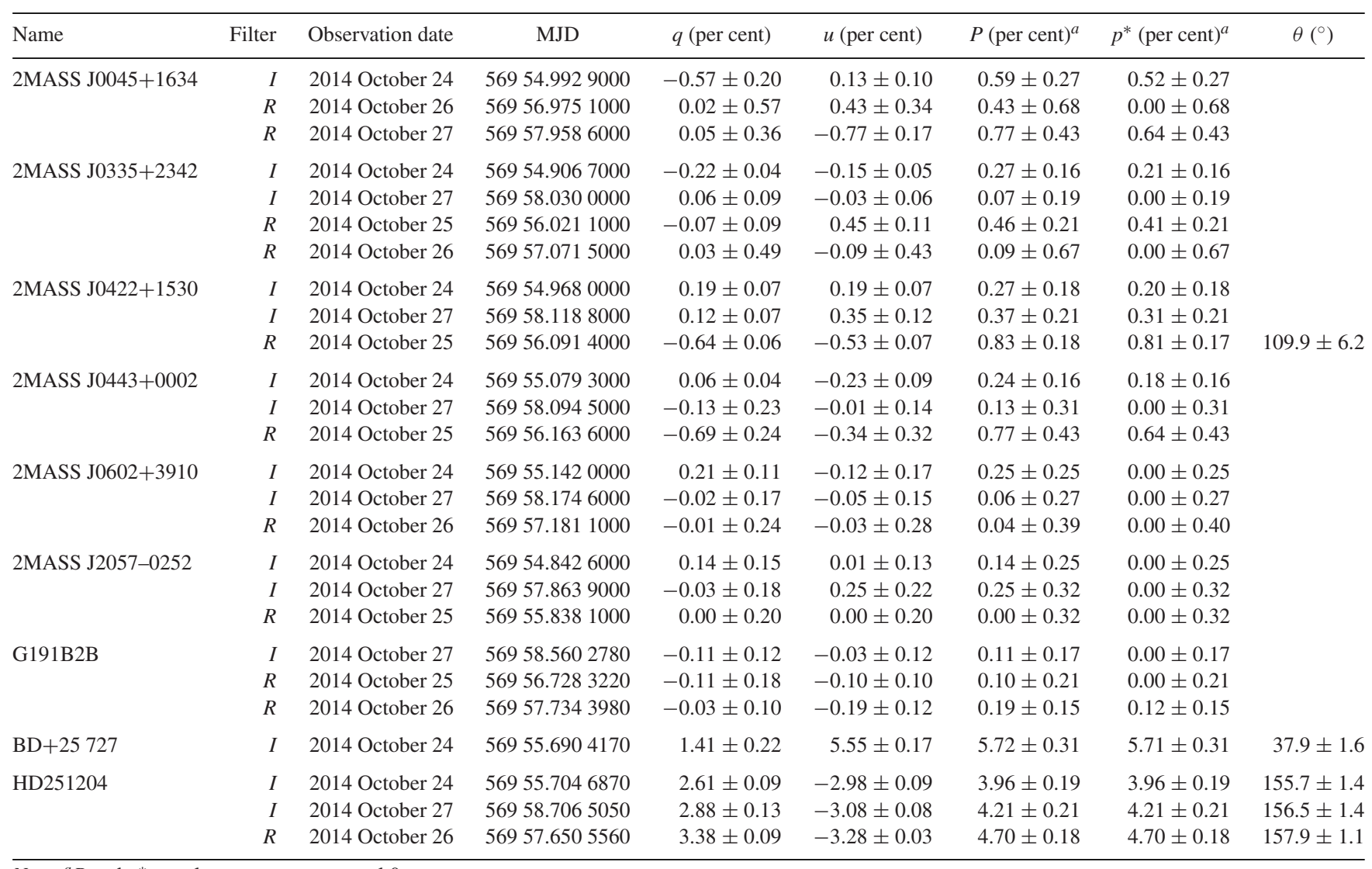

Note. ${ }^{a} P$ and $p^{*}$ are always greater or equal 0 .

\section{DISCUSSION}

All objects in our sample have a linear polarization degree in the $I$ band compatible with zero polarization. In the $R$ band, only $2 \mathrm{M} 0422$ has a value of linear polarization degree different from zero. The $3 \sigma$ upper limit on our detectability in the $I$ band is 0.69 per cent, and 1.0 per cent in the $R$ band. These upper limits were calculated by computing a mean of the uncertainties of all measurements of polarization for $I$ and $R$ bands, respectively, and multiplying by 3 the obtained values. Sengupta \& Marley (2010) predicted a maximum polarization of 0.8 per cent in the $I$ band for a $\log g=4.5$ object with $i=90^{\circ}$ and spectral type between M7 and L2. For a similar object but with $i=30^{\circ}$, the maximum predicted linear polarization is 0.2 per cent. Therefore, with the precision of our measurements, we would only be able to detect polarization for objects with $i$ close to $90^{\circ}$.

The positive detection of polarization on the $R$ band and not in the $I$ band might be due to the size of the particles producing the polarization, or to time variable polarization, depending on the mechanisms producing the polarization in $2 \mathrm{M} 0422$.

We describe the most plausible mechanisms that can explain polarization found in $2 \mathrm{M} 0422$ as follows:

(i) Interstellar dust in the line of sight between the Earth and the targets: Interstellar dust might be a significant source of polarization for objects at distances further than 100 pc (Tamburini, Ortolani \& Bianchini 2002; Bailey, Lucas \& Hough 2010; Cotton et al. 2016). If the distance derived by Faherty et al. (2012) is correct, then 2M0422 cannot be embedded in the Taurus-Auriga star-forming region, thus, interstellar dust would not be a plausible explanation for the detected polarization for 2M0422.

In the case in which the distance obtained by Liu et al. (2016) is correct, 2M0422 would be embedded on or behind the TaurusAuriga star-forming region. Therefore, the reddening found for $2 \mathrm{M} 0422$ colours and the detected polarization in the $R$ band, could be explained by the presence of interstellar dust. This kind of linear polarization has an exponential dependence with wavelength (Serkowski, Mathewson \& Ford 1975). The positive detection of strong polarization in the $R$ band and no significant polarization in the $I$ band, despite the proximity of the two central wavelengths, implies that the polarization is maximum at a wavelength $\leq 400 \mathrm{~nm}$, following the empirical function of the Serkowski's law of the interstellar medium polarization due to sub-micron particles (Serkowski et al. 1975; Whittet et al. 1992). We have adopted a $3 \sigma$ upper limit of $P(I)=0.6$ per cent, which is three times the uncertainty associated with the polarimetric measurement of this particular source (Table 2), and $K=0.75$ after equation 3 of Whittet et al. (1992).

(ii) Protoplanetary disc or debris disc surrounding our target: Protoplanetary discs are expected to have lifetimes of about $10 \mathrm{Myr}$ (Luhman \& Mamajek 2012). Some of the objects in our sample have estimated ages around $\sim 10 \mathrm{Myr}$. Thus, the linear polarization detected in some of these objects may be originated by a protoplanetary disc. Debris discs persist up to several Myr, but up to date, none has been confirmed around brown dwarfs.

In addition, in the case in which polarization is produced by a disc, we should find an excess of flux in the whole mid and/or far-infrared, depending on the type of disc we might find. In Fig. 2, we show the colour $J-W 2$ as a function of the near-infrared spectral type 


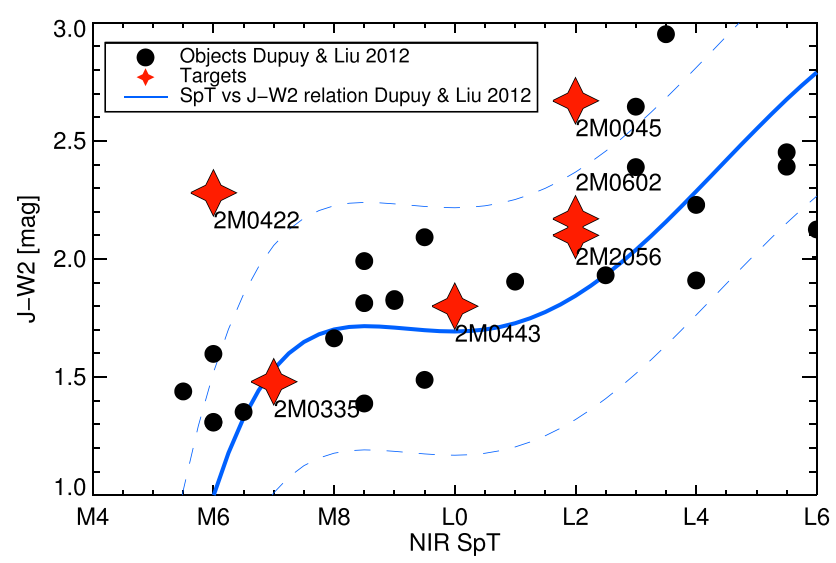

Figure 2. $J-W 2$ colour as a function of the near-infrared spectral type. Our targets are plotted as red stars, objects from Dupuy \& Liu (2012) are plot as black dots, the spectrophotometric relationship derived by the same authors is plotted as a blue thick line, and the $1 \sigma$ limits are plot as a dashed thin blue line. Uncertainties are smaller than the symbols. Objects $2 \mathrm{M} 0422$ and $2 \mathrm{M} 0045$ are above the $1 \sigma$ limit, indicating mid-infrared excess, and the possibility of the existence of a circumstellar disc.

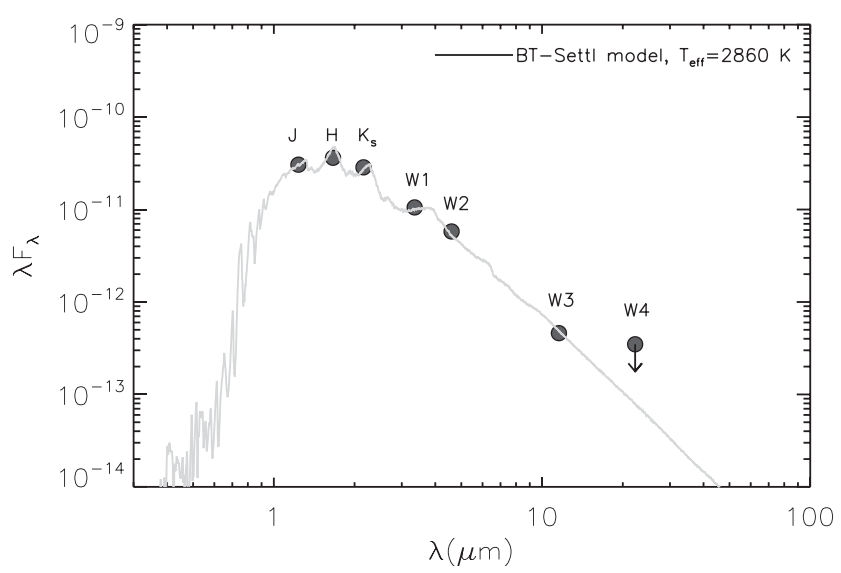

Figure 3. We fitted the 2MASS JHKs photometry of object 2M0422 to the BT-settl atmospheric model with $\mathrm{T}_{\text {eff }}=2860 \mathrm{~K}$ (this is the $\mathrm{T}_{\text {eff }}$ corresponding to a M6 dwarf using the conversion from spectral type to $\mathrm{T}_{\mathrm{eff}}$ from Herczeg \& Hillenbrand 2014). We employed two free parameters: the angular diameter $\theta$ of the source and the extinction $\left(A_{V}\right)$ in the $\mathrm{V}$ band. We assumed a radius for $2 \mathrm{M} 0422$ of $1 R_{J u p}$. We used the extinction law of Cardelli, Clayton \& Mathis (1989) to redden model atmospheres, and adopted a total to selective extinction value typical of interstellar medium dust $\left(R_{V}=3.1\right)$. The fit give a visual extinction for the object of $A_{v} \sim 3.1 \mathrm{mag}$.

of our targets, and we compared them to the colours of the objects in Dupuy \& Liu (2012), and the spectrophotometric relationship derived in the same work (blue line). Objects 2M J0422 and 2M J0045 are outside the $1 \sigma$ dispersion of the objects (dashed blue line), indicating mid-infrared flux excess in $J-W 2$ colour.

To try to confirm the existence of a disc, we searched for infrared excess in the whole spectral energy distribution (SED) of 2M0422 using data available in VizieR (Ochsenbein, Bauer \& Marcout 2000). After reddening the model atmospheric emission to fit its broadband photometry $\left(A_{V}=3.1 \mathrm{mag}\right)$, we fitted the SED predicted by the BT-Settl models for a M6 object, to the flux of 2M 0422+1530 in J, H, K (2MASS) and WISE (Fig. 3). No excess in the mid-infrared was found up to $11.6 \mu \mathrm{m}$. (iii) Presence of a transiting companion: During the transit, the symmetry of the dusty atmosphere of the young brown dwarf or giant exoplanet is broken, and the linear polarimetric signal should be enhanced (Sengupta 2016). The peak polarization is predicted to range between 0.1 and 0.3 per cent in the near-infrared. Sengupta (2016) estimated that the peak in polarization for $L$ dwarfs due to the transit of an Earth-size or larger exoplanets vary in the range between 0.2 and 1.0 per cent. Our data were not conceived as a continuous monitoring program. Therefore, this scenario, although possibly less plausible than disc extinction or dusty clouds, cannot be discarded for $2 \mathrm{M} 0422$.

(iv) Dust particles in the atmosphere of young brown dwarfs: L dwarf atmospheres are composed of clouds of iron and silicate grains of $\mathrm{H}_{2} \mathrm{O}, \mathrm{FeH}$ and $\mathrm{CO}$ (Cushing, Rayner \& Vacca 2005), which produces absorption bands in the near-infrared spectrum, and change the opacity of the atmosphere. The formation of the dust in ultracool dwarf atmospheres is influenced by surface gravity. Low surface gravity is expected to enhance the formation of dust in the atmospheres of these objects, and therefore a higher degree of linear polarization is expected for young brown dwarfs. As explained in Section 1, Marocco et al. (2014) and Hiranaka et al. (2016) concluded that under the scenario of dust haze of particles in the upper atmospheres, the grains must have sub-micron sizes (typically between 0.15 and $0.4 \mu \mathrm{m}$ ) in order to explain the observed red colours. Furthermore, that the polarization index is greater in the $R$ band than in the $I$ band in the M6 J0422+1530 also favours small characteristic particles, which better agrees with Hiranaka and Marocco et al.'s prediction. The detection of linear polarization in our targets would favour the scenario of the dusty atmospheres. The quite different polarimetric measurements in $R$ and $I$ band could be ascribed to time variable polarization intrinsic to $2 \mathrm{M} 0422$, as the data were acquired on different nights. This is the most plausible scenario if the parallax reported by Faherty et al. (2012) is correct.

\section{CONCLUSIONS}

In this work, we aimed to test the existence of optical linear polarization in a sample of brown dwarfs that show signs of youth characteristics in optical or near-infrared spectra. We selected the most extensive sample of young brown dwarfs observable from CAHA, that were bright enough in the $R$ and $I$ bands to achieve a precision of $\sigma_{P} \pm 0.2$ per cent in polarimetry observation with a 2.2-m telescope. We detected linear polarization for target 2MASS $\mathrm{J} 04221413+1530525$ in the $R$ band ( $p^{*}=0.81 \pm 0.17$ per cent). The two most plausible causes of the source of polarization in $2 \mathrm{M} 0422$ are

(i) Dust in the line of sight between the Earth and the object, only if we assume that the trigonometric parallax reported by Liu et al. (2016) is correct. In this case, 2M0422 might be embedded on or behind the Taurus-Auriga star-forming region. This hypothesis would explain the reddening of the spectrum of 2M0422, and the weak alkali lines indicating a young age of the object, in the case in which $2 \mathrm{M} 0422$ is a member of the star-forming region.

(ii) A protoplanetary or debris disc, or dust particles in the atmosphere of the object, if we assume Faherty et al. (2012) as correct. The last hypothesis would agree with Marocco et al. (2014) and Hiranaka et al. (2016) predictions about the sub-micron particles in young brown dwarf atmospheres, which would explain red colours and the detection of polarization in the $R$ band.

For some of the objects in which polarization was not detected, the cause might be that a polarimetric cycle may cover a significant 
fraction of the rotation. If polarization is caused by inhomogeneous dusty concentrations over the surface, then it might be diluted over long exposures, or it might be below our detection limits.

To confirm the cause of the detected linear polarization in young brown dwarfs and in brown dwarfs in general, further polarimetric data with higher precision are necessary in the optical and in the near-infrared.

\section{ACKNOWLEDGEMENTS}

We are thankful to the referee P. Lucas, for his valuable comments.

We gratefully acknowledge CAHA allocation time committee and CAHA Observatory staff for assiting the PI during the observations. This work was supported by Sonderforschungsbereich SFB 881 'The Milky Way System' (sub-project B6) of the German Research Foundation (DFG). This research has made use of the SIMBAD data base, operated at CDS, Strasbourg (France), and IRAF, the Image Reduction and Analysis Facility. This work was based on observations of the Calar Alto Observatory (Spain), using CAFOS at the $2.2 \mathrm{~m}$ telescope.

\section{REFERENCES}

Allers K. N., Liu M. C., 2013, ApJ, 772, 79

Allers K. N., Gallimore J. F., Liu M. C., Dupuy T. J., 2016, ApJ, 819, 133

Bailey J., Lucas P. W., Hough J. H., 2010, MNRAS, 405, 2570

Barman T. S., Macintosh B., Konopacky Q. M., Marois C., 2011, ApJ, 735, L39

Briceño C., Calvet N., Kenyon S., Hartmann L., 1999, AJ, 118, 1354

Burrows A. et al., 1997, ApJ, 491, 856

Cardelli J. A., Clayton G. C., Mathis J. S., 1989, ApJ, 345, 245

Cotton D. V., Bailey J., Kedziora-Chudczer L., Bott K., Lucas P. W., Hough J. H., Marshall J. P., 2016, MNRAS, 455, 1607

Cruz K. L., Reid I. N., Liebert J., Kirkpatrick J. D., Lowrance P. J., 2003, AJ, 126, 2421

Cruz K. L., Kirkpatrick J. D., Burgasser A. J., 2009, AJ, 137, 3345

Cushing M. C., Rayner J. T., Vacca W. D., 2005, ApJ, 623, 1115

Dupuy T. J., Liu M. C., 2012, ApJS, 201, 19

Faherty J. K. et al., 2012, ApJ, 752, 56

Faherty J. K. et al., 2016, ApJS, 225, 10

Gagné J., Lafrenière D., Doyon R., Malo L., Artigau É., 2014, ApJ, 783, 121

Gizis J. E., Monet D. G., Reid I. N., Kirkpatrick J. D., Liebert J., Williams R. J., 2000, AJ, 120, 1085

Gizis J. E. et al., 2012, AJ, 144, 94

Goldman B., Pitann J., Zapatero Osorio M. R., Bailer-Jones C. A. L., Béjar V. J. S., Caballero J. A., Henning T., 2009, A\&A, 502, 929

Gorlova N. I., Meyer M. R., Rieke G. H., Liebert J., 2003, ApJ, 593, 1074
Hawley S. L. et al., 2002, AJ, 123, 3409

Herczeg G. J., Hillenbrand L. A., 2014, ApJ, 786, 97

Hiranaka K., Cruz K. L., Douglas S. T., Marley M. S., Baldassare V. F., 2016, ApJ, 830, 96

Kendall T. R., Delfosse X., Martín E. L., Forveille T., 2004, A\&A, 416, L17

Kirkpatrick J. D. et al., 2012, ApJ, 753, 156

Lépine S., Shara M. M., Rich R. M., 2002, AJ, 124, 1190

Liu M. C., Dupuy T. J., Allers K. N., 2016, ApJ, 833, 96

Looper D. L. et al., 2008, ApJ, 686, 528

Lucas P. W., Roche P. F., Allard F., Hauschildt P. H., 2001, MNRAS, 326, 695

Luhman K. L., 2004, ApJ, 617, 1216

Luhman K. L., Mamajek E. E., 2012, ApJ, 758, 31

Marocco F. et al., 2014, MNRAS, 439, 372

Ménard F., Delfosse X., Monin J.-L., 2002, A\&A, 396, L35

Miles-Páez P. A., Zapatero Osorio M. R., Pallé E., Peña Ramírez K., 2013, A\&A, 556, A125

Miles-Páez P. A., Zapatero Osorio M. R., Pallé E., 2015, A\&A, 580, L12

Ochsenbein F., Bauer P., Marcout J., 2000, A\&AS, 143, 23

Patat F., Taubenberger S., 2011, A\&A, 529, A57

Reid I. N., Hawley S. L., Gizis J. E., 1995, AJ, 110, 1838

Reid I. N., Kirkpatrick J. D., Liebert J., Gizis J. E., Dahn C. C., Monet D. G., 2002, AJ, 124, 519

Seifahrt A., Reiners A., Almaghrbi K. A. M., Basri G., 2010, A\&A, 512, A37

Sengupta S., 2003, ApJ, 585, L155

Sengupta S., 2016, AJ, 152, 98

Sengupta S., Krishan V., 2001, ApJ, 561, L123

Sengupta S., Kwok S., 2005, ApJ, 625, 996

Sengupta S., Marley M. S., 2010, ApJ, 722, L142

Serkowski K., 1974, in Carleton N. P., ed., Polarization Techniques. p. 361

Serkowski K., Mathewson D. S., Ford V. L., 1975, ApJ, 196, 261

Shkolnik E., Liu M. C., Reid I. N., 2009, ApJ, 699, 649

Shkolnik E. L., Anglada-Escudé G., Liu M. C., Bowler B. P., Weinberger A. J., Boss A. P., Reid I. N., Tamura M., 2012, ApJ, 758, 56

Tamburini F., Ortolani S., Bianchini A., 2002, A\&A, 394, 675

Tata R., Martín E. L., Sengupta S., Phan-Bao N., Zapatero Osorio M. R., Bouy H., 2009, A\&A, 508, 1423

Wardle J. F. C., Kronberg P. P., 1974, ApJ, 194, 249

Whittet D. C. B., Martin P. G., Hough J. H., Rouse M. F., Bailey J. A., Axon D. J., 1992, ApJ, 386, 562

Zapatero Osorio M. R., Caballero J. A., Béjar V. J. S., 2005, ApJ, 621, 445

Zapatero Osorio M. R., Béjar V. J. S., Goldman B., Caballero J. A., Rebolo R., Acosta-Pulido J. A., Manchado A., Peña Ramírez K., 2011, ApJ, 740,4

Zapatero Osorio M. R., Béjar V. J. S., Miles-Páez P. A., Peña Ramírez K., Rebolo R., Pallé E., 2014, A\&A, 568, A6

\section{APPENDIX A}


Table A1. Observing log. Exposures format: exposure time $(\mathrm{s}) \times$ number of circles in angle $0^{\circ}$, exposure time $(\mathrm{s}) \times$ number of circles in angle $22^{\circ} .5$, exposure time $(\mathrm{s}) \times$ number of circles in angle $45^{\circ}$ and exposure time $(\mathrm{s}) \times$ number of circles in angle 67.5.

\begin{tabular}{|c|c|c|c|c|c|c|}
\hline Name & Date & Filter & Exposures & Airmass & Aperture $(\times$ FWHM $)$ & FWHM $(\operatorname{arcsec})$ \\
\hline \multirow{5}{*}{$2 \mathrm{M} 0045+1634$} & 2014 October 24 & $I$ & $120 \times 4,120 \times 4,120 \times 4,120 \times 4$ & 1.15 & $2-5$ & 2.3 \\
\hline & 2014 October 26 & $R$ & $300 \times 2,300 \times 2,300 \times 2,300 \times 2$ & 1.12 & $2-4$ & 1.5 \\
\hline & & & $360 \times 1,360 \times 1,360 \times 1,360 \times 1$ & & & 1.7 \\
\hline & 2014 October 27 & $R$ & $300 \times 2,300 \times 2,300 \times 2,300 \times 2$ & 1.07 & $2-4$ & 1.1 \\
\hline & & & $420 \times 1,420 \times 1,420 \times 1,420 \times 1$ & & & 1.4 \\
\hline \multirow[t]{7}{*}{$2 \mathrm{M} \mathrm{J} 0335+2342$} & 2014 October 23 & $R$ & $240 \times 1,240 \times 1,240 \times 1,240 \times 1$ & 1.04 & $2-4$ & 1.0 \\
\hline & & & $100 \times 2,100 \times 2,100 \times 2,100 \times 2$ & & & 1.2 \\
\hline & 2014 October 24 & $I$ & $100 \times 3,100 \times 3,100 \times 3,100 \times 3$ & 1.52 & $2-5$ & 1.3 \\
\hline & 2014 October 25 & $R$ & $240 \times 1,240 \times 1,240 \times 1,240$ & 1.03 & $2-4$ & 1.3 \\
\hline & & & $300 \times 1,300 \times 1,300 \times 1,300 \times 1$ & & & 1.3 \\
\hline & & & $260 \times 1,260 \times 1,260 \times 1,260 \times 1$ & & & 1.3 \\
\hline & 2014 October 26 & $R$ & $360 \times 3,360 \times 3,360 \times 3,360 \times 3$ & 1.05 & $2-4$ & 2.0 \\
\hline & 2014 October 27 & $I$ & $180 \times 1,180 \times 1,180 \times 1,180 \times 1$ & 1.17 & $2-5$ & 2.3 \\
\hline & & & $150 \times 3,150 \times 3,150 \times 3,150 \times 3$ & & & 2.3 \\
\hline \multirow{5}{*}{$2 \mathrm{M} \mathrm{J} 0443+0002$} & 2014 October 24 & $I$ & $150 \times 3,150 \times 3,150 \times 3,150 \times 3$ & 1.25 & $2-5$ & 3.4 \\
\hline & 2014 October 25 & $R$ & $300 \times 2,300 \times 2,300 \times 2,300 \times 2$ & 1.40 & $2-4$ & 1.1 \\
\hline & & & $310 \times 1,310 \times 1,310 \times 1,310 \times 1$ & & & 1.2 \\
\hline & 2014 October 27 & $I$ & $150 \times 1,150 \times 1,150 \times 1,150 \times 1$ & 1.25 & $2-5$ & 2.2 \\
\hline & & & $180 \times 1,180 \times 1,180 \times 1,180 \times 1$ & & & 2.3 \\
\hline \multirow[t]{3}{*}{$2 \mathrm{M} \mathrm{J} 0602+3910$} & 2014 October 24 & $I$ & $100 \times 4,100 \times 4,100 \times 4,100 \times 4$ & 1.00 & $2-5$ & 1.7 \\
\hline & 2014 October 26 & $R$ & $360 \times 3,360 \times 3,360 \times 3,360 \times 3$ & 1.02 & $2-4$ & 1.9 \\
\hline & 2014 October 27 & $I$ & $150 \times 3,150 \times 3,150 \times 3,150 \times 3$ & 1.10 & $2-5$ & 1.9 \\
\hline \multirow[t]{2}{*}{$2 \mathrm{M} \mathrm{J} 2057-0252$} & 2014 October 24 & $I$ & $120 \times 1,120 \times 1,120 \times 1,120 \times 1$ & 1.42 & $2-5$ & 3.9 \\
\hline & & & $150 \times 1,150 \times 1,150 \times 1,150 \times 1$ & & & 4.3 \\
\hline & 2014 October 26 & $R$ & $4 \times 3,4 \times 3,4 \times 3,4 \times 3$ & 1.19 & $2-6$ & 1.8 \\
\hline & 2014 October 27 & $I$ & $4 \times 1,4 \times 1,4 \times 1,4 \times 1$ & 1.06 & $2-6$ & 1.4 \\
\hline & & & $4.5 \times 1,4.5 \times 1,4.5 \times 1,4.5 \times 1$ & & & 1.4 \\
\hline & & & $5 \times 1,5 \times 1,5 \times 1,5 \times 1$ & & & 1.4 \\
\hline & 2014 October 27 & $R$ & $4 \times 3,4 \times 3,4 \times 3,4 \times 3$ & 1.06 & $2-6$ & 1.7 \\
\hline \multirow[t]{5}{*}{$\mathrm{BD}+284211^{a, c}$} & 2014 October 25 & $R$ & $1 \times 3,1 \times 3,1 \times 3,1 \times 3$ & 1.02 & $2-6$ & 1.2 \\
\hline & 2014 October 26 & $R$ & $1.5 \times 3,1.5 \times 3,1.5 \times 3,1.5 \times 3$ & 1.28 & $2-6$ & 1.6 \\
\hline & 2014 October 27 & $I$ & $2 \times 2,2 \times 2,2 \times 2,2 \times 2$ & 1.01 & $2-6$ & 1.1 \\
\hline & & & $2.5 \times 2,2.5 \times 2,2.5 \times 2,2.5 \times 2$ & & & 1.1 \\
\hline & 2014 October 27 & $R$ & $1.5 \times 3,1.5 \times 3,1.5 \times 3,1.5 \times 3$ & 1.01 & $2-6$ & 1.1 \\
\hline \multirow[t]{3}{*}{$\mathrm{BD}+25727^{b}$} & 2014 October 24 & $I$ & $0.1 \times 3,0.1 \times 3,0.1 \times 3,0.1 \times 3$ & 1.12 & $2-6$ & 1.2 \\
\hline & 2014 October 27 & $R$ & $0.5 \times 2,0.5 \times 2,0.5 \times 2,0.5 \times 2$ & 1.37 & $2-6$ & 2.9 \\
\hline & 2014 October 27 & $I$ & $0.4 \times 3,0.4 \times 3,0.4 \times 3,0.4 \times 3$ & 1.32 & $2-6$ & 2.1 \\
\hline \multirow[t]{4}{*}{ HD251204 ${ }^{b}$} & 2014 October 24 & $I$ & $0.8 \times 2,0.8 \times 2,0.8 \times 2,0.8 \times 2$ & 1.05 & $2-6$ & 1.1 \\
\hline & 2014 October 25 & $R$ & $0.8 \times 3,0.8 \times 3,0.8 \times 3,0.8 \times 3$ & 1.07 & $2-6$ & 2.2 \\
\hline & 2014 October 26 & $R$ & $2 \times 3,2 \times 3,2 \times 3,2 \times 3$ & 1.03 & $2-6$ & 2.2 \\
\hline & 2014 October 27 & $I$ & $0.9 \times 3,0.9 \times 3,0.9 \times 3,0.9 \times 3$ & 1.06 & $2-6$ & 2.2 \\
\hline
\end{tabular}

Notes. ${ }^{a}$ Non-polarized standard star. ${ }^{b}$ Highly polarized standard star. ${ }^{c} \mathrm{BD}+284211$ was not used as calibration star due to the presence of a nearby companion that may introduced spurious polarization.

This paper has been typeset from a $\mathrm{T}_{\mathrm{E}} \mathrm{X} / \mathrm{L} \mathrm{T}_{\mathrm{E}} \mathrm{X}$ file prepared by the author. 